\title{
Evaluation of "Diabetes in Pregnancy Study Group of India" (DIPSI) Criterion as a Diagnostic Test for Gestational Diabetes Mellitus
}

\author{
Aradhana Singh", Raj K Singh ${ }^{2}$, Vani Aditya ${ }^{3}$, Priyanka Singh ${ }^{4}$ \\ ${ }^{1}$ Associate Professor, Dept. of Obstetrics and Gynaecology, BRD Medical College, Gorakhpur, UP, India. \\ ${ }^{2}$ Associate Professor, Dept. of Medicine, BRD Medical College, Gorakhpur, UP, India. \\ ${ }^{3}$ Professor \& Head of Dept. of Obstetrics and Gynaecology, BRD Medical College, Gorakhpur, UP, India. \\ ${ }^{4}$ Assistant Professor, Dept. of Obstetrics and Gynaecology, BRD Medical College, Gorakhpur, UP, India. \\ Corresponding Author: Aradhana Singh
}

\begin{abstract}
Introduction: In view of the alarmingly increasing incidence of Gestational Diabetes Mellitus (GDM), worldwide, as well as in India, a consensus to bring about standardization for diagnosis of GDM is needed. Internationally, the WHO 2013 criteria is being widely used, while (Diabetes In Pregnancy Study Group of India) DIPSI non fasting criteria is popularly used in India. There still remains many missed cases of GDM in India, which is a cause of concern. This study was done to compare the DIPSI non fasting, with WHO 2013 criteria for diagnosis of GDM.

Methods: this cross-sectional study included 530 pregnant women, attending antenatal clinic of a tertiary care center, of North India, from Feb. 2018 to March 2019 and fulfilling inclusion criteria. All women attending antenatal clinic, during the study period, were subjected to capillary glucose evaluation, 2 hours after $75 \mathrm{~g}$ oral glucose load, irrespective of the timing of last meal (DIPSI), as part of routine antenatal checkup. After 7 days, only those 530 women, who turned up in fasting state, and fulfilled inclusion criteria, were enrolled in the study and subjected to fasting $75 \mathrm{~g}$, oral glucose tolerance test (OGTT) (WHO 2013). Accuracy of DIPSI test was compared, to the fasting WHO 2013 criteria. Capillary glucose was measured using regular, well calibrated, point of care, "Optium Free Style" glucometer.

Results: Out of 107 women diagnosed to have GDM by WHO 2013 criteria, only 89 were diagnosed by the DIPSI criteria. DIPSI had a low sensitivity (83.18\%) when compared to the WHO 2013 criteria,

Conclusion: This study showed that when non fasting DIPSI criteria was used as diagnostic criteria, $10.58 \%$ women with GDM, missed the diagnosis. Considering the adverse maternal and perinatal outcome of GDM, implication of missed diagnosis would be grave, especially in a developing country, like India. Hence, use of DIPSI criteria for diagnosing GDM should be reconsidered.
\end{abstract}

Key words: GDM, DIPSI, OGTT, Diabetes in Pregnancy.

\section{INTRODUCTION}

The prevalence of Gestational diabetes mellitus (GDM), that is diabetes diagnosed during pregnancy, is increasing alarmingly in India. It is estimated that about 4 million pregnant women are affected with GDM in India, at any given time point. ${ }^{1}$
GDM is the most common metabolic disorder of pregnancy and is associated with increased maternal, fetal and neonatal morbidity and mortality. ${ }^{2}$ The repercussion of diabetes in pregnancy, is not only on the current generation but also on the next generation. Evidences suggest that infants of mothers with diabetes in pregnancy are at 
increased risk of obesity, diabetes, hypertension and T2DM, later in life. Mothers, with history of GDM are at increased risk of T2DM and cardiovascular disease. $^{3,4,5}$ Keeping in view, the rising trend, if cases of GDM are missed, our coming generation would be crippled with diabetes and metabolic syndrome and would constitute a population with suboptimal health and poor productivity.

Screening and early diagnosis of diabetes in pregnancy, may prevent or reduce both immediate and long-term harm, to mother and baby. ${ }^{6}$

Hence, this disease should be given utmost priority and rigorous research for a sensitive screening modality and its uniform and universal implementation should be initiated.

Unfortunately, in India, despite increase in antenatal care and institutional deliveries, there still remains many missed cases of GDM, amounting to a great harm to public health in our country.

After the establishment of DIPSI criteria as non-fasting screening test for GDM, it is being widely used in India, for the sake of simplicity and better compliance. ${ }^{7}$ This criterion is considered appropriate for a developing country like India, because of uncertainty of follow up visits due to lack of awareness and illiteracy still prevailing at large in our country.

Internationally, the WHO 2013 criteria, of fasting oral glucose tolerance test (OGTT), is used to diagnose GDM and is considered gold standard. ${ }^{8}$

Many studies from different parts of our country have shown poor sensitivity of DIPSI for diagnosis of GDM.

This study was aimed at evaluating the sensitivity of DIPSI criteria in comparison to the internationally accepted WHO 2013 criteria for diagnosis of GDM.

\section{MATERIAL AND METHODS}

This cross-sectional observational study included 530 pregnant women, attending antenatal OPD of a tertiary care center of North India. Pregnant women attending antenatal OPD, from Feb. 2018 to March 2019 underwent capillary glucose evaluation, 2 hours after $75 \mathrm{~g}$ oral glucose load, irrespective of their timing of last meal, as part of routine antenatal checkup. They were called after an overnight fast of at least 8 hours, after 7 days, for their next visit. 530 Pregnant women fulfilling inclusion criteria and giving consent and who turned up in fasting state, were subjected to 1 hour and 2-hour oral $75 \mathrm{~g}$ glucose, capillary glucose evaluation, according to the WHO 2013 criteria. Detailed history and physical examination were performed and BMI was calculated according to pre pregnancy or first trimester weight. All procedures followed were in accordance with the ethical standards.

Accuracy of DIPSI was compared with fasting $75 \mathrm{~g}$ OGTT, using cut offs, as per the 2013 WHO criteria, for diagnosis of GDM. Capillary glucose was measured using regular, well calibrated, point of care, "Optium Freestyle" glucometer, in the antenatal OPD.

Known diabetics, critically ill pregnant women, pregnant women taking medications affecting glycemic control (steroids, beta mimetics etc.) were excluded from the study.

For comparison between groups, Pearson's Chi Square test was used. A p-value of < 0.05 was set as the significance threshold.

\section{RESULTS}

Table 1: Patient characteristics of GDM positive patients

\begin{tabular}{|l|l|l|l|c|c|}
\hline Patient Characteristics & Total = 530 & \multicolumn{2}{l|}{ GDM positive (n= 107) } & Chi square & P value \\
\hline Age (years) & & $\begin{array}{l}\text { DIPSI positive } \\
(\mathbf{n = 8 9})\end{array}$ & $\begin{array}{l}\text { WHO (2013) positive } \\
(\mathbf{n = 1 0 7})\end{array}$ & & \\
\hline $18-24$ & $159(30 \%)$ & $35(39.3 \%)$ & $42(39.3 \%)$ & & \\
\hline $25-34$ & $275(51.9 \%)$ & $51(57.3 \%)$ & $62(57.9 \%)$ & 0.0546 & .973 \\
\hline$\geq 35$ & $96(18.1 \%)$ & $03(3.4 \%)$ & $03(2.8 \%)$ & & \\
\hline
\end{tabular}


Aradhana Singh et.al. Evaluation of "diabetes in pregnancy study group of India" (DIPSI) criterion as a diagnostic test for gestational diabetes mellitus.

\begin{tabular}{|c|c|c|c|c|c|}
\hline \multicolumn{6}{|c|}{ Table 1 : Continued... } \\
\hline \multicolumn{6}{|c|}{ Gestational age (weeks) } \\
\hline$\leq 12$ & $126(23.8 \%)$ & $04(4.5 \%)$ & $07(6.5 \%)$ & & \\
\hline $13-26$ & $195(36.8 \%)$ & $28(31.5 \%)$ & $34(31.8 \%)$ & 0.4077 & .8155 \\
\hline $27-40$ & $219(41.3 \%)$ & $57(64.0 \%)$ & $66(74.1 \%)$ & & \\
\hline \multicolumn{6}{|c|}{ BMI $\left(\mathrm{kg} / \mathrm{m}^{2}\right)$} \\
\hline $18.5-24.9$ & $335(63.2 \%)$ & $38(42.7 \%)$ & $39(36.4 \%)$ & & \\
\hline $25-29.9$ & $138(26.0 \%)$ & $49(55.1 \%)$ & $63(58.9 \%)$ & 1.407 & .494 \\
\hline $30-34.9$ & $57(10.8 \%)$ & $02(2.2 \%)$ & $05(4.7 \%)$ & & \\
\hline
\end{tabular}

Table 2: Cross analysis of diagnostic capability of DIPSI with WHO 2013 criteria

\begin{tabular}{|l|l|l|}
\hline GDM as diagnosed by & \multicolumn{2}{|l|}{ DIPSI test result } \\
\cline { 2 - 3 } WHO 2013 criterion & Positive & Negative \\
\hline Positive $(\mathrm{n}=107)$ & $89(83.18 \%)$ & $18(16.82 \%)$ \\
\hline Negative $(\mathrm{n}=423)$ & 0 & $423(100 \%)$ \\
\hline Total $=530$ & $89(16.79 \%)$ & $441(83.21 \%)$ \\
\hline
\end{tabular}

Table 3: Statistical parameter of DIPSI with WHO 2013 criteria.

\begin{tabular}{|l|l|}
\hline Statistical parameter & Value \\
\hline Sensitivity & $83.18 \%$ \\
\hline Specificity & $100 \%$ \\
\hline Positive predictive value & $100 \%$ \\
\hline Negative predictive value & $96.07 \%$ \\
\hline
\end{tabular}

\section{DISCUSSION}

A total of 530 pregnant women, who were successfully followed up, were included in the study. 107 women $(20.18 \%)$ were diagnosed to have GDM by the 2013 WHO criteria, while only 89 women (16.79\%) were diagnosed to have GDM by DIPSI criteria.

The DIPSI non-fasting 75g, 2 hours cut off of $140 \mathrm{mg} / \mathrm{dl}$, had a low sensitivity of $83.18 \%$, when compared to the 2013 WHO criteria. 18 pregnant women $(16.82 \%)$ with GDM, missed diagnosis by DIPSI criteria. These missed cases were falsely labelled as normal, probably because, the test did not account for fasting hyperglycemia. All pregnant women diagnosed with nonfasting DIPSI criteria were also diagnosed as GDM with fasting 2013 WHO criteria. Thus, DIPSI had $100 \%$ positive predictive value. However, its negative predictive value was poor $(96.07 \%)$.

Considering the prevalence of 5 million GDM cases per year in our country, ${ }^{9}$ this would translate into approximately 8 lakhs and 41 thousand missed GDM cases per year, which is a huge numbered to be ignored.

There is high quality evidence indicating that, early diagnosis and treatment of GDM statistically significantly reduces the relative risk of adverse outcome in both, the mother and the baby. ${ }^{10}$ Missing the diagnosis would have grave consequences over two successive generations.

Many comparative studies from different parts of our country have shown poor sensitivity of the DIPSI criterion. ${ }^{11}$ In the largest study till date, in urban and rural Tamil Nadu, as part of Indian Diabetes Federation sponsored WINGS program, DIPSI was found to have poor sensitivity of $27.7 \%$ when compared to the WHO 1999 criteria and $22.6 \%$ when compared to the (International Association of Diabetes and Pregnancy study Groups) IADPG criteria. ${ }^{12}$

Another study from New Delhi, showed that $22.36 \%$ GDM women missed the diagnosis when DIPSI criteria was used. ${ }^{13}$ However, a few studies have shown higher sensitivity of DIPSI. ${ }^{14,15}$

In a study by Ghada $M$ et al in Egypt, sensitivity and specificity of DIPSI was $100 \%$ and $98.5 \%$ respectively, as compared to IADPG. But positive predictive value was only $83 \%$ and negative predictive value was $100 \%$. The author predicted that higher sensitivity of DIPSI may be due to the diurnal variation in insulin level, glucose tolerance and beta cell responsiveness, taking into consideration that DIPSI could be performed at any time in the day while WHO test could be done in the morning. ${ }^{16}$

DIPSI is being widely used in India, for screening and diagnosis of GDM, for the sake of ease and better compliance. In recent years, India has been successful in reaching health care facility to rural and remote areas, through primary health care centers and sub centers and through mobile medical units. If the test facility is made available to the smallest and the most remote health care facilities, getting pregnant women in fasting state or testing 
made available to their doorsteps, would not be a great challenge.

The evidence base of DIPSI criteria is a single center study, comparing nonfasting OGTT with the WHO 1999 criteria, showing $100 \%$ sensitivity and specificity. ${ }^{17}$

Hyperglycemia and adverse pregnancy outcome (HAPO) derived and IADPG consensus panel formulated, diagnostic criteria, has been adopted and endorsed by the WHO in 2013. This criterion for GDM diagnosis is based, not on the diagnostic accuracy, but on the prognostic accuracy, as derived from the HAPO study based, risk of adverse neonatal outcome. The IADPG consensus panel, decided the diagnostic threshold for fasting, 1 hour and 2 hours plasma glucose concentration, at which the key pregnancy outcomes, i.e., birth weight $>90^{\text {th }}$ percentile, cord C Peptide $>90^{\text {th }}$ percentile and neonatal percent body fat $>90^{\text {th }}$ percentile, are increased 1.75 folds over the mean HAPO study population. Adjustments were also made for race and ethnic groups, parity, age, BMI, smoking and alcohol use, presence or absence of family history of diabetes etc.

Large outcome-based studies comparing DIPSI with WHO criteria is needed.

\section{CONCLUSIONS}

WHO in 2013, adopted the IADPG criteria, for diagnosis of GDM, because this is the only outcome-based criteria and has the ability to diagnose and treat GDM earlier, thereby reducing maternal, fetal and neonatal complications. DIPSI is easy and simple, but has lower sensitivity as compared to the WHO 2013 criterion and implementing the WHO criterion universally in India, should be considered.

\section{Acknowledgement: None}

\section{Conflict of Interest: None}

Source of Funding: None

Ethical Approval: Approved

\section{REFERENCES}

1. Mithal A, Bansal B, Kalra S. Gestational diabetes in India: Science and society. Indian J Endocr Metab 2015; 19:701-4.

2. Martis R, Crowther CA, Shepherd E, Alsweiler J, Downie MR, Brown J. treatments for women with gestational diabetes mellitus: an overview of Cochrane systematic reviews. 2018, Issue 8. Art. No. CD012327. DOI:10.1002/14651858. CD012327.pub2.

3. Bellamy L, Casas JP, Hingorani AD, Williams D. Type 2 diabetes mellitus after gestational diabetes: A systematic review and meta-analysis. Lancet.2009;373(9677):1773-9.

4. Retnakaran R, Qi Y, Connelly PW, Sermer M, Zinman B, Hanley AJ. Glucose intolerance in pregnancy and postpartum risk of metabolic syndrome in young women. J Clin Endocrinol Metab.2010;95(2):670-7

5. Shah BR, Retnakaran R, Booth GL. Increased risk of cardiovascular disease in young women following gestational diabetes mellitus. Diabetes Care. 2008;31(8):1668-9.

6. International association of diabetes and pregnancy study groups consensus panel, Metzger BE, Gabbe SG, Persson B, et al. IADPSG recommendations on the diagnosis and classification of hyperglycemia in pregnancy. Diabetes care 2010; 33:676-82.

7. Government of India. Maternal and Health Division, Diagnosis and Management of Gestational Diabetes Mellitus: Technical and Operational Guidelines. New Delhi, India: Ministry of Health and Family Welfare, New Concept Information System; 2018.

8. Diagnostic criteria and classification of hyperglycemia first detected in pregnancy. Geneva: World Health Organization; 2013 (WHO/NMH/MND/13.2;

http://www.who.int/diabetes/publications/hy perglycemia_In_Pregnancy/en/)

9. International Diabetes Federation. IDF Diabetes Atlas, $8^{\text {th }}$ edn. Brussels, Belgium: International Diabetes Federation, 2017.

10. Crowther CA, Hiller JE, Moss JR, McPhee AJ, Jeffries WS, Robinson JS; Australian Carbohydrate Intolerance Study in Pregnant Women (ACHOIS) Trial Group. Effect of treatment of gestational diabetes mellitus on 
Aradhana Singh et.al. Evaluation of "diabetes in pregnancy study group of India" (DIPSI) criterion as a diagnostic test for gestational diabetes mellitus.

pregnancy outcomes. N Engl J Med 2005; 352:2477-86.

11. Tripathi R, Verma D, Gupta VK, Tyagi S, Kalaivani M, Ramji S, Mala YM. Evaluation of $75 \mathrm{~g}$ glucose load in nonfasting state [Diabetes in Pregnancy Study group of India (DIPSI) criteria] as a diagnostic test for gestational diabetes mellitus. Indian J Med Res. 2017 Feb; 145(2): 209-214. doi: 10.4103/ijmr.IJMR_1716_15. PMID: 28639597; PMCID: PMC5501053.

12. Mohan V, Mahalakshmi MM, Bhavadharini B, Maheshwari K, Kalaiyarasi G, Anjana RM et al. Comparison of screening for gestational diabetes mellitus by oral glucose tolerance tests done in non-fasting and fasting states. Acta Diabetol. 2014 Dec.51(6):1007-13. Doi: 10.1007/s00592014-0660-5.Epub 2014 Oct 15.

13. Pulkit Vij, Sujeet Jha SK, Gupta, Aneja Anjila, Mathur Rajani, Waghdhare Swati et al. Comparison of DIPSI and IADPG criteria for diagnosis of GDM: A study in North Indian tertiary care center. Int $\mathbf{J}$ Diabetes Dev Ctries. 2015:1-4.

14. Geetha N, Gowri KS. Comparison of Iadpsg And Dipsi Criteria for Diagnosis of
Gestational Diabetes Mellitus. IOSR-JDMS 2016; 15: 1-4.

15. Rudra S, Yadav A. Efficacy of Diabetes in Pregnancy Study Group India as a Diagnostic Tool for Gestational Diabetes Mellitus in a Rural Setup in North India. J South Asian Feder Obs Gynae 2019; 11 (6):349-352.

16. El Sagheer, G.M., Hamdi, L. Prevalence and risk factors for gestational diabetes mellitus according to the Diabetes in Pregnancy Study Group India in comparison to International Association of the Diabetes and Pregnancy Study Groups in El-Minya, Egypt. Egypt J Intern Med 30, 131-139 (2018).

https://doi.org/10.4103/ejim.ejim_11_18.

17. Seshiah V, Balaji V, Balaji MS, Sanjeevi CB, Green A. Gestational diabetes mellitus in India. J Assoc Physicians India. 2004 Sep; 52:707-11. PMID: 15839447

How to cite this article: Singh A, Singh RK, Aditya V et.al. Evaluation of "diabetes in pregnancy study group of India" (DIPSI) criterion as a diagnostic test for gestational diabetes mellitus. Int J Health Sci Res. 2021; 11(6):1-5. DOI: https://doi.org/10.52403/ijhsr. 20210601 